\title{
Real fixed points and singular values of two-parameter family $\lambda \frac{z}{\left(e^{z}-1\right)^{n}}$
}

\author{
Mohammad Sajid \\ College of Engineering, Qassim University, Buraidah, Al-Qassim, Saudi Arabia
}

Received: 18 January 2016, Accepted: 12 December 2016

Published online: 23 January 2017.

\begin{abstract}
The two-parameter family of $f_{\lambda, n}(z)=\lambda \frac{z}{\left(e^{z}-1\right)^{n}}, \lambda \in \mathbb{R} \backslash\{0\}, z \in \mathbb{C} \backslash\{0\}, n \in \mathbb{N} \backslash\{1\}$, is considered in this paper. The existence and nature of the real fixed points of $f_{\lambda, n}(x), x \in \mathbb{R} \backslash\{0\}$ are described for $n$ odd and $n$ even. It is found that the function $f_{\lambda, n}(z)$ possesses infinitely many singular values. It has also been shown that some critical values of $f_{\lambda, n}(z)$ lie in the closure and other lie into the exterior of the disk centered at origin and having radius $\lambda$.
\end{abstract}

Keywords: Fixed point, critical value, singular values.

\section{Introduction}

The fixed points and singular values are generally very important to study the behavior of dynamical systems. The real dynamics of functions is mostly centered around fixed points and has become an interesting research area, partially due to applicability of it for describing properties of the Julia sets and Fatou sets [1,5,6,9,15]. Some advanced results on transcendental dynamics can be seen in [8]. The real dynamics of the cubic polynomials and generalized logistic maps are investigated in [4] and [7] respectively. The real fixed points of one parameter families of functions are found in [3, $10,11]$ and the real fixed points of two-parameter family are described in [2]. The singular values of one parameter families are shown in [12,13] and the singular values of two-parameter family are found in [14].

Let $f: \mathbb{C} \rightarrow \mathbb{C}$. A point $z^{*} \in \mathbb{C}$ is said to be a critical point of $f(z)$ if $f^{\prime}\left(z^{*}\right)=0$. The value $f\left(z^{*}\right)$ corresponding to a critical point $z^{*}$ is called a critical value of $f(z)$. A point $w \in \hat{\mathbb{C}}=\mathbb{C} \cup\{\infty\}$ is said to be an asymptotic value for $f(z)$, if there exists a continuous curve $\gamma:[0, \infty) \rightarrow \widehat{\mathbb{C}}$ satisfying $\lim _{t \rightarrow \infty} \gamma(t)=\infty$ and $\lim _{t \rightarrow \infty} f(\gamma(t))=w$. A singular value of $f$ is defined to be either a critical value or an asymptotic value of $f$. A point $x$ is said to be a fixed point of function $f(x)$ if $f(x)=x$. A fixed point $x_{f}$ is called an attracting, neutral (indifferent) or repelling if $\left|f^{\prime}\left(x_{f}\right)\right|<1,\left|f^{\prime}\left(x_{f}\right)\right|=1$ or $\left|f^{\prime}\left(x_{f}\right)\right|>1$ respectively.

Let

$$
\mathscr{F}=\left\{f_{\lambda, n}(z)=\lambda \frac{z}{\left(e^{z}-1\right)^{n}}: \lambda \in \mathbb{R} \backslash\{0\}, z \in \mathbb{C} \backslash\{0\}, n \in \mathbb{N} \backslash\{1\}\right\}
$$

be two-parameter family of transcendental meromorphic functions which is neither even nor odd. For $n=1$, the singular values and fixed points of $\lambda \frac{z}{e^{z}-1}$ are already investigated in [11].

The present paper describes the fixed points and singular values of $f_{\lambda, n} \in \mathscr{F}$. In Theorem 1 and Theorem 2 , the existence 
and nature of real fixed points of $f_{\lambda, n}(x)$ are investigated for $n$ odd and $n$ even respectively. It is found that the function $f_{\lambda, n} \in \mathscr{F}$ has infinitely many singular values in Theorem 3. It is seen that, in Theorem 4, the function $f_{\lambda, n}^{\prime}(z)$ has no zeros in the right half plane. In Theorem 5, the function $f_{\lambda, n}(z)$ maps the left half in the closure and into the exterior of the disk according to two different regions respectively, centered at origin and having radius $\lambda$. Further, in Theorem 6 , it is shown that some critical values of $f_{\lambda, n} \in \mathscr{F}$ lie in the closure and other lie into the exterior of the disk centered at origin and having radius $\lambda$.

Lemma 1. Let $g(x)=1-\frac{n x}{1-e^{-x}}, x \in \mathbb{R}, n \in \mathbb{N} \backslash\{1\}$. Then, $\lim _{x \rightarrow-\infty} g(x)=1, g(0)=1-n, \lim _{x \rightarrow+\infty} g(x)=-\infty, g\left(x^{*}\right)=-1$ and $g\left(x^{* *}\right)=-1$, where $x^{*}$ and $x^{* *}$ are the unique negative real roots of the equation $(2-n x) e^{x}-2=0$ for odd $n \in \mathbb{N} \backslash\{1\}$ and even $n \in \mathbb{N} \backslash\{1\}$ respectively.

Proof. It is easily seen that

$$
\begin{gathered}
\lim _{x \rightarrow-\infty} g(x)=1-n \lim _{x \rightarrow-\infty} \frac{x}{1-e^{-x}}=1-n \times 0=1 \\
g(0)=1-n \lim _{x \rightarrow 0} \frac{x}{1-e^{-x}}=1-n \times 1=1-n \\
\lim _{x \rightarrow+\infty} g(x)=1-n \lim _{x \rightarrow+\infty} \frac{x}{1-e^{-x}}=1-n \times \infty=-\infty \\
g\left(x^{*}\right)=1-\frac{n x^{*}}{1-e^{-x^{*}}}=1-\frac{n x^{*}}{n x^{*} / 2}=-1 \text { since } 1-e^{-x^{*}}=n x^{*} / 2 \\
g\left(x^{* *}\right)=1-\frac{n x^{* *}}{1-e^{-x^{* *}}}=1-\frac{n x^{* *}}{n x^{* *} / 2}=-1 \text { since } 1-e^{-x^{* *}}=n x^{* *} / 2 .
\end{gathered}
$$

This completes the proof of lemma.

\section{Real fixed points of $f_{\lambda, n} \in \mathscr{F}$ and their nature}

Let us define, for $n$ odd,

$$
\lambda^{*}=\frac{x^{*}}{f\left(x^{*}\right)}=\left(e^{x^{*}}-1\right)^{n}
$$

where $x^{*}$ is given in Lemma 1 .

The following theorem gives the nature of real fixed points of the function $f_{\lambda, n} \in \mathscr{F}$ for $n$ odd greater than 1:

Theorem 1. Let $f_{\lambda, n} \in \mathscr{F}$ for odd $n \in \mathbb{N} \backslash\{1\}$.

(a) The function $f_{\lambda, n}(x)$ has a unique real fixed point $x_{\lambda}$ for $\lambda>-1$ and no real fixed point for $\lambda<-1$. The fixed point $x_{\lambda}$ of $f_{\lambda, n}(x)$ is negative for $-1<\lambda<0$ and is positive for $\lambda>0$.

(b) The fixed point $x_{\lambda}$ of $f_{\lambda, n}(x)$ is attracting for $-1<\lambda<0$ and $0<\lambda<\lambda^{*}$, rationally indifferent for $\lambda=\lambda^{*}$ and repelling for $\lambda>\lambda^{*}$.

Proof.

(a) For fixed points of $f_{\lambda, n}(x), \lambda \frac{x}{\left(e^{x}-1\right)^{n}}=x$. For $n$ odd greater than 1 , it gives a unique fixed point $x_{\lambda}=\ln \left(1+\lambda^{1 / n}\right)$. Therefore, the fixed point $x_{\lambda}$ of $f_{\lambda, n}(x)$ is real for $\lambda>-1$ and no real fixed point for $\lambda<-1$. Consequently, the real fixed point $x_{\lambda}$ of $f_{\lambda, n}(x)$ is positive if $\lambda>0$ and negative if $-1<\lambda<0$.

(b) Since $x_{\lambda}$ is a fixed point of $f_{\lambda, n}(x)$, then

$$
f_{\lambda, n}^{\prime}\left(x_{\lambda}\right)=\lambda \frac{\left(1-n x_{\lambda}\right) e^{x_{\lambda}}-1}{\left(e^{x_{\lambda}}-1\right)^{n+1}}
$$


Substituting $\lambda=\left(e^{x} \lambda-1\right)^{n}$ in Equation (1), we get

$$
f_{\lambda, n}^{\prime}\left(x_{\lambda}\right)=\left(e^{x_{\lambda}}-1\right)^{n} \frac{\left(1-n x_{\lambda}\right) e^{x_{\lambda}}-1}{\left(e^{x_{\lambda}}-1\right)^{n+1}}=1-\frac{n x_{\lambda}}{1-e^{-x_{\lambda}}} .
$$

It is easily seen that, using Lemma $1,-1<f_{\lambda, n}^{\prime}\left(x_{\lambda}\right)<1$ for $-1<\lambda<0$. Hence, the fixed point $x_{\lambda}$ of $f_{\lambda, n}(x)$ is attracting for $-1<\lambda<0$.

Again, by Lemma 1 , it is found that $-1<f_{\lambda, n}^{\prime}\left(x_{\lambda}\right)<1$ for $0<\lambda<\lambda^{*}, f_{\lambda, n}^{\prime}\left(x_{\lambda}\right)=-1$ for $\lambda=\lambda^{*}$ and $f_{\lambda, n}^{\prime}\left(x_{\lambda}\right)<-1$ for $\lambda>\lambda^{*}$. Therefore, the fixed point $x_{\lambda}$ of $f_{\lambda, n}(x)$ is attracting for $0<\lambda<\lambda^{*}$, rationally indifferent for $\lambda=\lambda^{*}$ and repelling for $\lambda>\lambda^{*}$.

This completes the proof of theorem.

Remark. The fixed point $x_{\lambda}$ of $f_{\lambda, n}(x)$ is increasing if $\lambda$ increases.

Let us define, for $n$ even,

$$
\lambda^{* *}=\frac{x^{* *}}{f\left(x^{* *}\right)}=\left(e^{x^{* *}}-1\right)^{n}
$$

where $x^{* *}$ is given in Lemma 1.

For $n$ even greater than 0 , the description of the fixed points $f_{\lambda, n} \in \mathscr{F}$ are different than for $n$ odd greater than 1 . The following theorem shows the nature of real fixed points of the function $f_{\lambda, n} \in \mathscr{F}$ for $n$ even greater than 0 ,

Theorem 2.Let $f_{\lambda, n} \in \mathscr{F}$ for even $n \in \mathbb{N} \backslash\{1\}$.

(i) The function $f_{\lambda, n}(x)$ has two real fixed points for $0<\lambda<1$, a unique real fixed point for $\lambda \geq 1$ and no real fixed point for $\lambda<0$. One fixed point $x_{\lambda}^{-}$is negative for $0<\lambda<1$ and other fixed point $x_{\lambda}^{+}$is positive for $0<\lambda<1$ and for $\lambda \geq 1$.

(ii) The fixed point $x_{\lambda}^{-}$is attracting for $0<\lambda<1$ and the fixed point $x_{\lambda}^{+}$is attracting for $0<\lambda<\lambda^{* *}$, rationally indifferent for $\lambda=\lambda^{* *}$ and repelling for $\lambda>\lambda^{* *}$.

Proof.

(i) For fixed points of $f_{\lambda, n}(x), \lambda \frac{x}{\left(e^{x}-1\right)^{n}}=x$. For even $n \in \mathbb{N} \backslash\{1\}$, the fixed points are given as $x_{\lambda}=\ln \left(1 \pm \lambda^{1 / n}\right)$. It follows that $f_{\lambda, n}(x)$ has two real fixed points for $0<\lambda<1$, a unique real fixed point for $\lambda \geq 1$ and no real fixed point for $\lambda<0$. For $0<\lambda<1$, it is easily seen that one fixed point $x_{\lambda}^{-}$is negative and other fixed point $x_{\lambda}^{+}$is positive. For $\lambda \geq 1$, the real fixed point $x_{\lambda}^{+}$of $f_{\lambda, n}(x)$ is positive.

(ii) Since $x_{\lambda}$ is a fixed point of $f_{\lambda, n}(x)$ and $\lambda=\left(e^{x_{\lambda}}-1\right)^{n}$, then we have

$$
f_{\lambda, n}^{\prime}\left(x_{\lambda}\right)=\left(e^{x_{\lambda}}-1\right)^{n} \frac{\left(1-n x_{\lambda}\right) e^{x_{\lambda}}-1}{\left(e^{x_{\lambda}}-1\right)^{n+1}}=1-\frac{n x_{\lambda}}{1-e^{-x_{\lambda}}} .
$$

By Lemma 1, it is easily found that $-1<f_{\lambda, n}^{\prime}\left(x_{\lambda}^{-}\right)<1$ for $0<\lambda<1$ and $-1<f_{\lambda, n}^{\prime}\left(x_{\lambda}^{+}\right)$for $0<\lambda<\lambda^{* *}$. It follows that the fixed point $x_{\lambda}^{-}$is attracting for $0<\lambda<1$ and the fixed point $x_{\lambda}^{+}$is attracting for $0<\lambda<\lambda^{* *}$.

Again, using Lemma $1, f_{\lambda, n}^{\prime}\left(x_{\lambda}^{+}\right)=-1$ for $\lambda=\lambda^{* *}$ and $f_{\lambda, n}^{\prime}\left(x_{\lambda}^{+}\right)<-1$ for $\lambda>\lambda^{* *}$. Therefore, the fixed point $x_{\lambda}^{+}$ of $f_{\lambda, n}(x)$ is rationally indifferent for $\lambda=\lambda^{* *}$ and is repelling for $\lambda>\lambda^{* *}$.

The proof of theorem is completed. 


\section{Singular values of $f_{\lambda, n} \in \mathscr{F}$}

The following theorem shows that the function $f_{\lambda, n} \in \mathscr{F}$ has infinitely many singular values,

Theorem 3. Let $f_{\lambda, n} \in \mathscr{F}$. Then, the function $f_{\lambda, n}(z)$ possesses infinitely many singular values.

Proof. For critical points, $f_{\lambda, n}^{\prime}(z)=\lambda \frac{\left(e^{z}-1\right)^{n}-n z\left(e^{z}-1\right)^{n-1} e^{z}}{\left(e^{z}-1\right)^{2 n}}=0$. It gives $(n z-1) e^{z}+1=0$. Using the real and imaginary parts of this equation, we have

$$
\begin{gathered}
\frac{y}{\sin y}-\frac{1}{n} e^{y \cot y-\frac{1}{n}}=0 \\
x=\frac{1}{n}-y \cot y
\end{gathered}
$$

It is seen that, from Figure 1, Equation (2) has infinitely many solutions since intersections on horizontal axis are increased if interval increases on it.

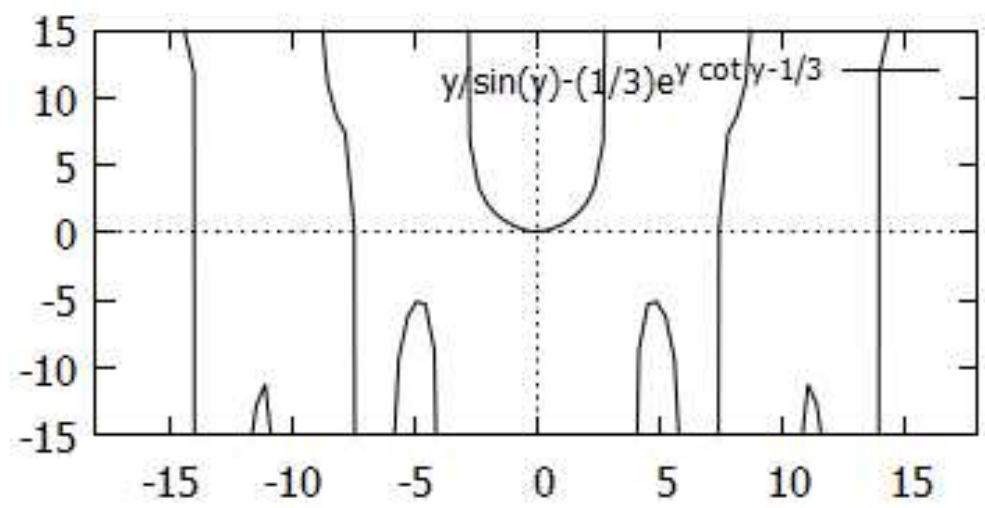

Fig. 1: Graph of $\frac{y}{\sin y}-\frac{1}{n} e^{y \cot y-\frac{1}{n}}$ for $n=3$

Suppose $\left\{y_{k}\right\}_{k=-\infty, k \neq 0}^{k=\infty}$ are solutions of Equation (2). From Equation (3), $x_{k}=\frac{1}{n}-y_{k} \cot y_{k}$ for $k= \pm 1, \pm 2, \pm 3, \ldots$ Consequently, it gives that $z_{k}=x_{k}+i y_{k}$ are critical points of $f_{\lambda, n}(z)$ since $f_{\lambda}^{\prime}\left(z_{k}\right)=0$, and then the critical values are given by $f_{\lambda, n}\left(z_{k}\right)$. It is observed that $f_{\lambda, n}\left(z_{k}\right)$ are distinct for different $k$. It follows that $f_{\lambda, n} \in \mathscr{F}$ has infinitely many critical values.

The finite asymptotic value of $f_{\lambda, n}(z)$ is 0 since $f_{\lambda, n}(z) \rightarrow 0$ as $z \rightarrow \infty$ along positive real axis.

Thus, it shows that $f_{\lambda, n} \in \mathscr{F}$ possesses infinitely many singular values.

Remark. For the zeros of $f_{\lambda, n}^{\prime}(z)$ on imaginary axis, from Equation (4), it is found that $\cos y-i \sin y=1-i n y$. This equation gives $y=0$. Therefore, $f_{\lambda, n}^{\prime}(z)$ has no zeros on imaginary axis.

Let $H^{+}=\{z \in \hat{\mathbb{C}}: \operatorname{Re}(z)>0\}$ be the right half plane. The following theorem gives that the function $f_{\lambda, n}^{\prime}(z)$ has no zeros in the right half plane $H^{+}$:

Theorem 4.Let $f_{\lambda, n} \in \mathscr{F}$. Then, the function $f_{\lambda, n}^{\prime}(z)$ has no zeros in $H^{+}$. 
Proof. Suppose $\operatorname{Re}(z)>0$, and $f_{\lambda, n}^{\prime}(z)=\lambda \frac{(1-n z) e^{z}-1}{\left(e^{z}-1\right)^{n+1}}=0$ which implies that $e^{-z}=1-n z$. Then,

$$
\frac{\cos y-i \sin y}{e^{x}}=1-n x-i n y
$$

Firstly, when $y \neq 0$, then, by imaginary part of Equation (4), $\frac{\sin y}{y}=n e^{x}>1$. This is not true for $y>0$ and for $y<0$ because $\frac{\sin y}{y}$ is an even function.

Secondly, when $y=0$, then $z=x>0$ and, by real part of Equation (4), $e^{x}=\frac{1}{1-n x}$. For $x>\frac{1}{2}$ and $n \geq 2$, it is not valid because the left hand side is positive and the right hand side is negative. It is obviously not true when $x=\frac{1}{2}$. This is also false for $0<x<\frac{1}{2}$ since it is easily observed that, by Figure 2, there is no any intersection of the function $e^{x}$ with the function $\frac{1}{1-n x}$ for $n=2,3,4,5, \ldots$.

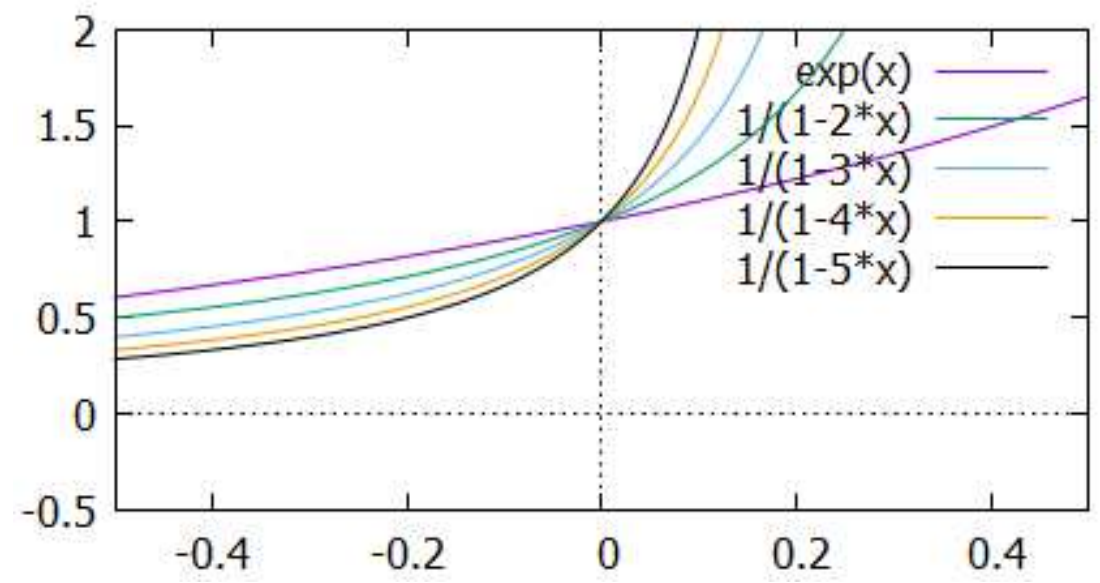

Fig. 2: Graphs of $e^{x}$ and $\frac{1}{1-n x}$ for $n=2,3,4,5$

Thus, it is concluded that the function $f_{\lambda, n}^{\prime}(z)$ has no zeros in $H^{+}$.

Let $H^{-}=\{z \in \hat{\mathbb{C}}: \operatorname{Re}(z)<0\}$ be the left half plane. In the following theorem, it is shown that the function $f_{\lambda, n} \in \mathscr{F}$ maps the left half plane in the closure and into the exterior of the disk according to two different regions respectively.

Theorem 5. Let $f_{\lambda, n} \in \mathscr{F}$. Then, the function $f_{\lambda, n}(z)$ maps $H^{-}$in the closure and into the exterior of the disk centered at origin and having radius $\lambda$ for $|z| \geq 1$ and $|z|<1$ respectively.

Proof. Consider the line segment $\gamma$ which is defined by $\gamma(t)=t z, t \in[0,1]$ and suppose the function $h(z)=e^{z}$ for an arbitrary fixed $z \in H^{-}$. Then

$$
\int_{\gamma} h(z) d z=\int_{0}^{1} h(\gamma(t)) \gamma^{\prime}(t) d t=z \int_{0}^{1} e^{t z} d t=e^{z}-1
$$

Since $M \equiv \max _{t \in[0,1]}|h(\gamma(t))|=\max _{t \in[0,1]}\left|e^{t z}\right|<1$ and $m \equiv \min _{t \in[0,1]}|h(\gamma(t))|=\min _{t \in[0,1]}\left|e^{t z}\right|>\varepsilon>0$ for $z \in H^{-}$, then

$$
m|z|<|z| \leq\left|e^{z}-1\right|=\left|\int_{\gamma} h(z) d z\right| \leq M|z|<|z|
$$

Using the left side of Inequality (5), $\left|\frac{z}{\left(e^{z}-1\right)^{n}}\right| \leq \frac{1}{|z|^{n-1}} \leq 1$ for all $|z| \geq 1$ and $n \in \mathbb{N} \backslash\{1\}$. Hence, $\left|f_{\lambda, n}(z)\right|=\left|\lambda \frac{z}{\left(e^{z}-1\right)^{n}}\right| \leq|\lambda|$ for all $|z| \geq 1$. It gives that the function $f_{\lambda, n}(z)$ maps $H^{-}$in the closure of the disk centered at origin and having radius 
$\lambda$ for all $|z| \geq 1$. Similarly, using the right side of Inequality (5), $\left|\frac{z}{\left(e^{z}-1\right)^{n}}\right|>\frac{1}{|z|^{n-1}}>1$ for all $|z|<1$ and $n \in \mathbb{N} \backslash\{1\}$. Therefore, it follows that $\left|f_{\lambda, n}(z)\right|=\left|\lambda \frac{z}{\left(e^{z}-1\right)^{n}}\right|>|\lambda|$ for all $|z|<1$. This shows that the function $f_{\lambda, n}(z)$ maps $H^{-}$into the exterior of the disk centered at origin and having radius $\lambda$ for all $|z|<1$.

The following theorem proves that the function $f_{\lambda, n} \in \mathscr{F}$ has some critical values in the closure and other into the exterior of the disk centered at origin and having radius $\lambda$ according to two different regions respectively.

Theorem 6. Let $f_{\lambda, n} \in \mathscr{F}$. Then, some critical values of $f_{\lambda, n}(z)$ lie in the closure and other into the exterior of the disk centered at origin and having radius $\lambda$ for $z \geq 1$ and $z<1$ in the left half plane respectively.

Proof. All the critical points of $f_{\lambda, n}(z)$ lie in $H^{-}$since, by Theorem $4, f_{\lambda, n}^{\prime}(z)$ has no zeros in $H^{+}$. Moreover, by Theorem 5 , the function $f_{\lambda, n}(z)$ maps the left half plane $H^{-}$in the closure and into the exterior of the disk centered at origin and having radius $\lambda$ for $|z| \geq 1$ and $|z|<1$ respectively. Therefore, it follows that some critical values of $f_{\lambda, n}(z)$ lie in the closure and other into the exterior of the disk centered at origin and having radius $\lambda$ for $z \geq 1$ and $z<1$ in the left half plane respectively.

\section{Conclusion}

This paper is generalization of one parameter family $\lambda \frac{z}{e^{z}-1}$ as two-parameter family $\lambda \frac{z}{\left(e^{z}-1\right)^{n}}$. The real fixed points and singular values of two-parameter family of functions $f_{\lambda, n}(z)=\lambda \frac{z}{\left(e^{z}-1\right)^{n}}, \lambda \in \mathbb{R} \backslash\{0\}, z \in \mathbb{C} \backslash\{0\}, n \in \mathbb{N} \backslash\{1\}$, have investigated. It is seen that the real fixed points of $f_{\lambda, n}(z)$ have different nature for $n$ odd and $n$ even. We have found that the function $f_{\lambda, n}(z)$ has infinitely many singular values for $n \in \mathbb{N} \backslash\{1\}$. We have also shown that some critical values of $f_{\lambda, n}(z)$ lie in the closure and other into the exterior of the open disk centered at origin and having radius $\lambda$ according to two different regions in the left half plane.

\section{Competing interests}

The authors declare that they have no competing interests.

\section{Authors' contributions}

All authors have contributed to all parts of the article. All authors read and approved the final manuscript.

\section{References}

[1] G. P. Kapoor and M. G. P. Prasad. Dynamics of $\left(e^{z}-1\right) / z$ : the Julia set and bifurcation. Ergodic Theory and Dynamical Systems, 18(6):1363-1383, 1998. http://dx.doi.org/10.1017/S0143385798118011

[2] M. G. Lee and C. C. Ho. Fixed points of two-parameter family of function $\lambda\left(\frac{x}{b^{x}-1}\right)^{n}$. Applied Mathematics, 6(3):576-584, 2015. http://dx.doi.org/10.4236/am.2015.63052

[3] D. Lim. Fixed points and dynamics on generating function of Genocchi numbers. J. Nonlinear Sci. Appl., 9(3):933-939, 2016.

[4] A. A. Magrenan and J. M. Gutierrez. Real dynamics for damped Newton's method applied to cubic polynomials. Journal of Computational and Applied Mathematics, 275:527-538, 2015. http://dx.doi.org/10.1016/j.cam.2013.11.019

[5] T. Nayak and M. G. P. Prasad. Julia sets of Joukowski-Exponential maps. Complex Anal. Oper. Theory, 8(5):1061-1076, 2014. http://dx.doi.org/10.1007/s11785-013-0335-1

[6] M. G. P. Prasad and T. Nayak. Dynamics of $\left\{\lambda \tanh \left(e^{z}\right): \lambda \in \mathbb{R} \backslash\{0\}\right\}$. Discrete and Continuous Dynamical Systems, 19(1):121138, September 2007. http://dx.doi.org/10.3934/dcds.2007.19.121 
[7] A. G. Radwan. On some generalized discrete logistic maps. J. Adv. Res., 4(2):163-171, 2013. http://dx.doi.org/10.1016/j.jare.2012.05.003

[8] P. J. Rippon and G. M. Stallard. Transcendental Dynamics and Complex Analysis. London Mathematical Society Lecture Note Series, 348, Cambridge University Press, 2008.

[9] M. Sajid. Real and complex dynamics of one parameter family of meromorphic functions. Far East Journal of Dynamical Systems, 19(2):89-105, 2012.

[10] M. Sajid. On real fixed points of one parameter family of function $\frac{x}{b^{x}-1}$. Tamkang Journal of Mathematics., 46(1):61-65, 2015. http://dx.doi.org/10.5556/j.tkjm.45.2014.1577

[11] M. Sajid. Singular values and fixed points of family of generating function of Bernoulli's numbers. J. Nonlinear Sci. Appl., 8(1):17-22, 2015.

[12] M. Sajid. Singular values of one parameter family $\lambda\left(\frac{z}{e^{z}-1}\right)^{m}$. International Mathematical Forum, 10(7):301-304, 2015. http://dx.doi.org/10.12988/imf.2015.5319.

[13] M. Sajid. Singular values of one parameter family of generalized generating function of Bernoulli's numbers. Appl. Math. Inf. Sci., 9(6):2921-2924, 2015. http://dx.doi.org/10.12785/amis/090619

[14] M. Sajid. Singular values of two parameter families $\lambda \frac{e^{a z}-1}{z}$ and $\lambda \frac{z}{e^{a z}-1}$. Journal of Mathematics Research, 8(1):10-13, 2016. http://dx.doi.org/10.5539/jmr.v8n1p10

[15] M. Sajid and G. P. Kapoor. Dynamics of transcendental meromorphic functions $\frac{\left(z+\mu_{0}\right)}{\left(z+\mu_{0}+4\right)} e^{z}$ having rational Schwarzian derivative. Dynamical systems: An International Journal, 22(3):323-337, September 2007. http://dx.doi.org/10.1080/14689360701208131 